\title{
Editorial
}

\section{Biologically Inspired Robotics 2016}

\author{
Liwei Shi $\mathbb{D}^{1},{ }^{1}$ Maki Habib, ${ }^{2}$ Nan Xiao $\mathbb{D},{ }^{1,3}$ and Huosheng $\mathrm{Hu}^{4}$ \\ ${ }^{1}$ The Institute of Advanced Biomedical Engineering System, School of Life Science, Beijing Institute of Technology, No. 5, \\ Zhongguancun South Street, Haidian District, Beijing 100081, China \\ ${ }^{2}$ School of Sciences and Engineering, The American University in Cairo, Cairo, Egypt \\ ${ }^{3}$ Faculty of Engineering, Kagawa University, 2217-20 Hayashi-cho, Takamatsu, Kagawa, Japan \\ ${ }^{4}$ School of Computer Science \& Electronic Engineering, University of Essex, Colchester, UK \\ Correspondence should be addressed to Liwei Shi; shiliwei@bit.edu.cn
}

Received 3 April 2018; Accepted 3 April 2018; Published 1 August 2018

Copyright (C) 2018 Liwei Shi et al. This is an open access article distributed under the Creative Commons Attribution License, which permits unrestricted use, distribution, and reproduction in any medium, provided the original work is properly cited.

Nature's evolution has introduced highly efficient biological functions, material, and mechanisms. Understanding the engineering principles in nature and how these evolved over time have inspired new forms of development. Bioinspiration establishes the basis of bioinspired design that offers an enormous potential for the improvement of all aspects of modern human life. This field emerges as an innovative research development field aiming to draw inspiration and substances from nature and create biological systems in functions, structure, and mechanism. This field introduces promising interdisciplinary scientific and technological challenges that are helping to solve modern human problems.

In every scientific development including the field of biologically inspired robotics, biological phenomena and ideas are inspiring innovations leading to new designs and development of new products and devices that include sensing, control, intelligence, behaviors, actuation, and mechanisms.

This special issue includes 5 papers and it is focused on the theoretical and technological challenges of evolutionary transformation from biological systems to intelligent robots.

The paper "ARM-Cortex M3-Based Two-Wheel Robot for Assessing Grid Cell Model of Medial Entorhinal Cortex: Progress towards Building Robots with Biologically Inspired Navigation-Cognitive Maps" by J. Cuneo et al. presents the implementation and use of a two-wheel autonomous robot and its effectiveness as a tool for studying the use of grid cells as part of mammalian's brains space-mapping circuitry. The paper introduces a discrete-time algorithm that emulates the medial entorhinal cortex and demonstrated it on the robot to explore a limited laboratory area. Position coordinate neural maps were achieved as mathematically predicted although for a reduced number of implemented neurons.

The paper "Design of a New Nonlinear Stiffness Compliant Actuator and its Error Compensation Method" by S. Lan and Z. Song discloses a design method for nonlinear stiffness compliant actuator that can perform a predefined deflection-torque trajectory of the regular phenomenon. A roller and a cantilever which have special curve profile constituted the basic mechanical structure of the nonlinear stiffness compliant actuators. An error compensation method was proposed to analyze the stiffness of elastic structure. The developed design was tested using simulation and the results show its effectiveness.

The paper "Review of Neurobiological Based Mobile Robot Navigation System Research Performed Since 2000" by P. Zeno et al. presents a literature survey of the state-of-the-art research on rodent neurobiological and neurophysiological based navigation systems that incorporate models of spatial awareness and navigation brain cells. The main focus was to explore the functionality of the cognitive maps developed in the mobile robot systems with respect to route planning, as well as a discussion/analysis of the computational complexity required to scale these systems.

The paper "Bioinspired Knee Joint for a Power-Assist Suit" by T. Kikuchi et al. proposes a bioinspired knee joint and torque adjustment mechanism to assist the standing movement of a human. The developed mechanism was evaluated with human subject for its motion, torque characteristics, and stress. Despite the $33 \%$ error in deep flexion, the measured torque was less than 120 degrees and fitted to the 
designed torque curve. The result demonstrated $30 \%$ and $63 \%$ reduction with the assistance from 100 -degree and 80 -degree knee angles, respectively.

The paper "A Review on Compliant Joint Mechanisms for Lower-Limb Exoskeletons" by M. Gálvez-Zúñiga and A. Aceves-López is focused on compliant joint mechanisms for lower limb aiming to comply with its user since 2000.

Liwei Shi Maki Habib

Nan Xiao

Huosheng $\mathrm{Hu}$ 


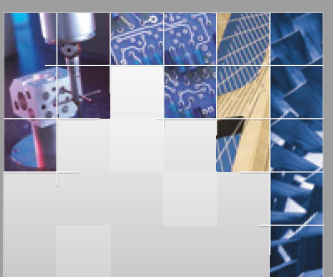

\section{Enfincering}
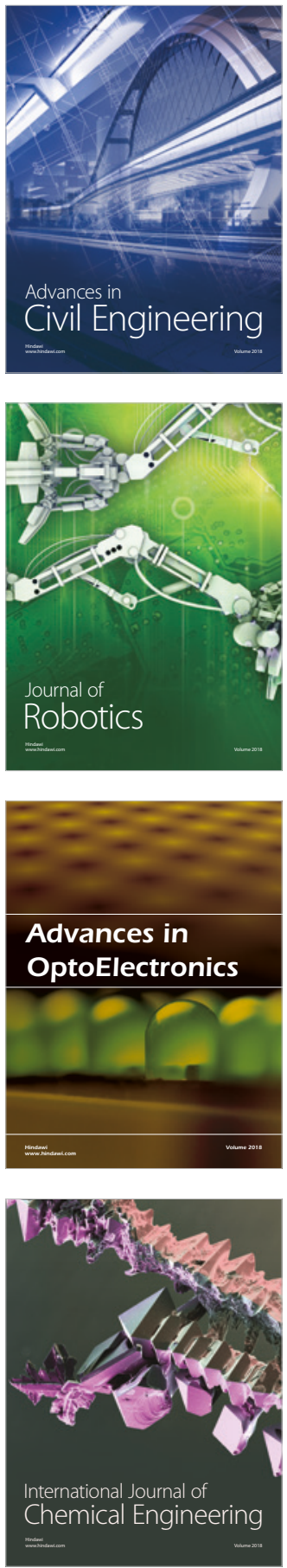

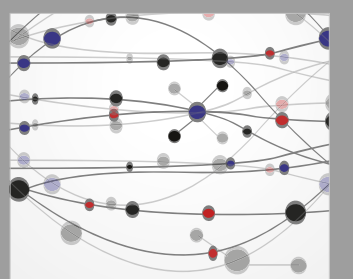

\section{Rotating \\ Machinery}

The Scientific World Journal

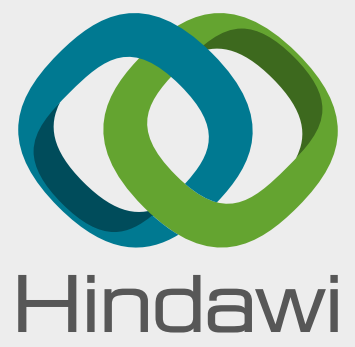

Submit your manuscripts at

www.hindawi.com
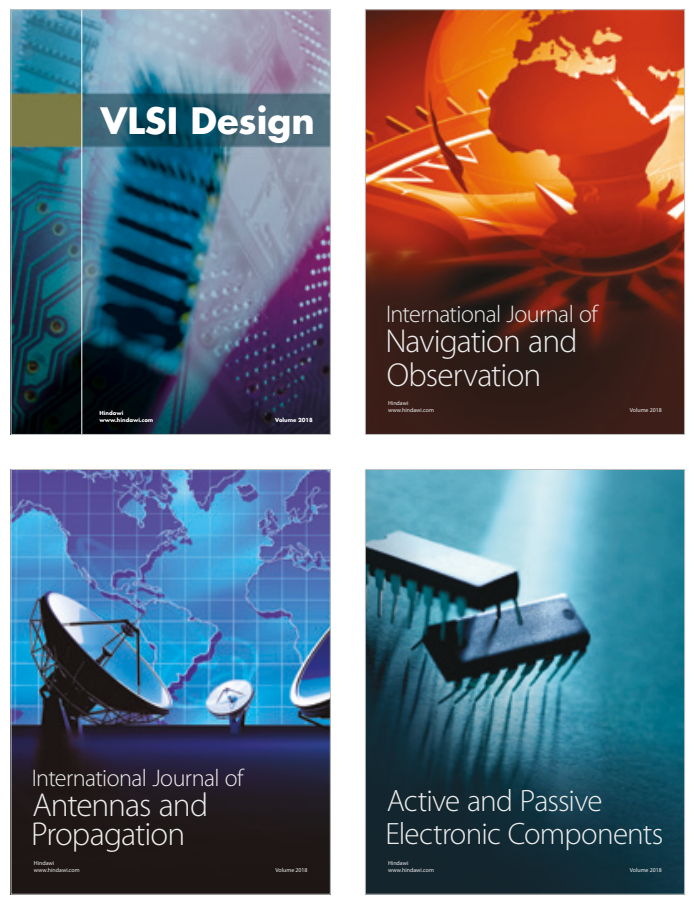
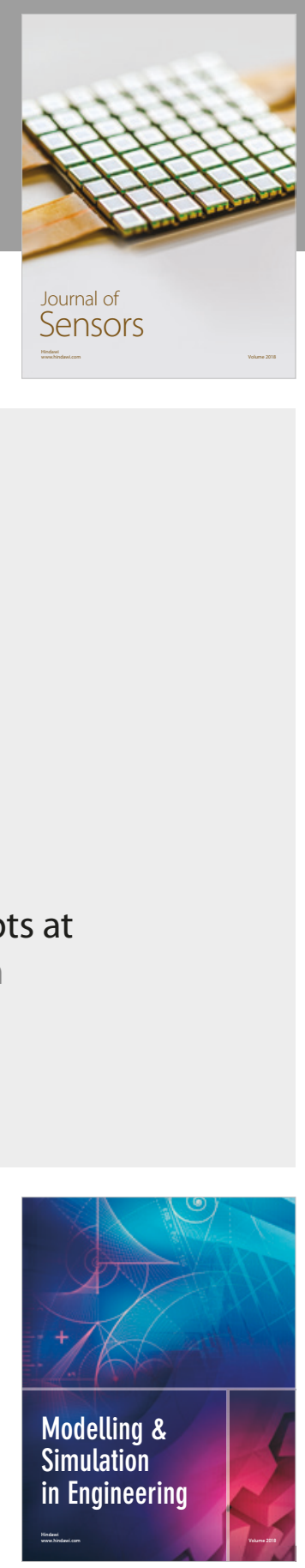

\section{Advances \\ Multimedia}
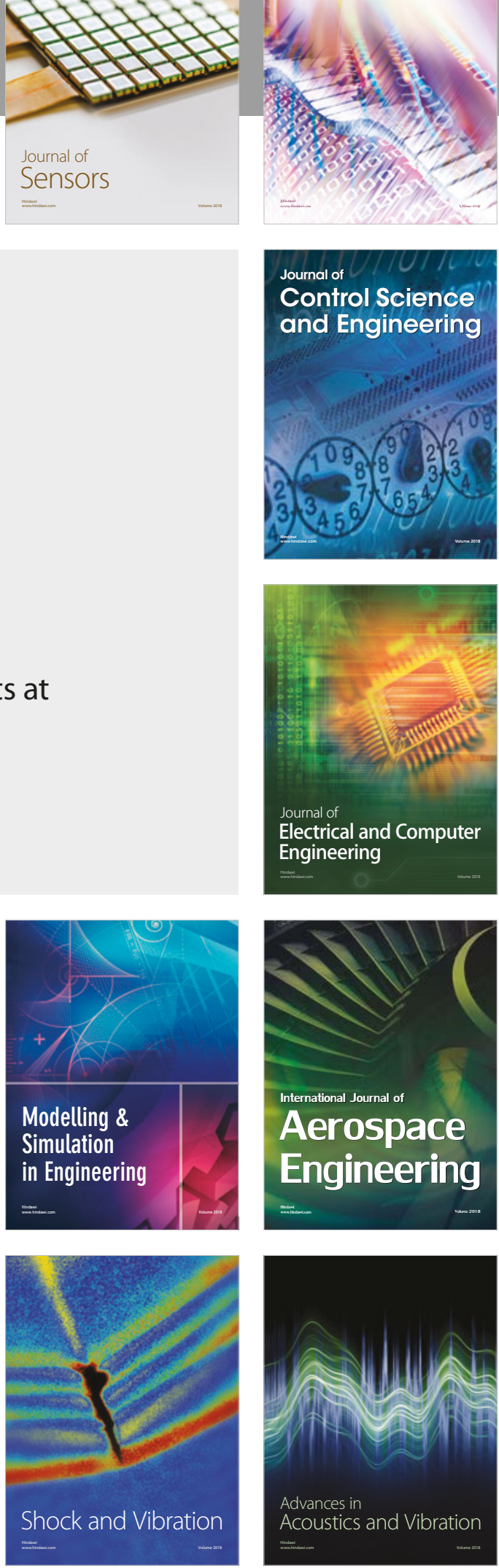